\title{
Cluster Partition Method of Large-Scale Grid-Connected Distributed Generations considering Expanded Dynamic Time Scenarios
}

\author{
Chang Ye, ${ }^{1}$ Kan Cao, ${ }^{1}$ Haiteng Han $\mathbb{D},{ }^{2}$ Ziwen Liu $\mathbb{D},{ }^{2}$ Defu Cai, ${ }^{1}$ and Dan Liu ${ }^{1}$ \\ ${ }^{1}$ State Grid Hubei Electric Power Co., Ltd., Wuhan 430077, China \\ ${ }^{2}$ College of Energy and Electrical Engineering, Hohai University, Nanjing, 210098, China \\ Correspondence should be addressed to Haiteng Han; hanht@hhu.edu.cn
}

Received 18 September 2021; Revised 8 December 2021; Accepted 9 January 2022; Published 16 February 2022

Academic Editor: Dazhong Ma

Copyright (c) 2022 Chang Ye et al. This is an open access article distributed under the Creative Commons Attribution License, which permits unrestricted use, distribution, and reproduction in any medium, provided the original work is properly cited.

\begin{abstract}
The reasonable clustering of large-scale distributed generations (DGs) can optimize the scheduling control and operation monitoring of the power grid, which ensures the orderly and efficient integration of DGs into the power system. In this article, the influence of internal and external flexible resources is considered in the DG cluster partition, and the comprehensive performance indexes with expanded dynamic time scenario are proposed to realize the dynamic cluster partition. Firstly, the active and reactive power balance indexes considering the flexible resources are derived, which forms the comprehensive index together with the structure index. Then, the comprehensive index is expanded to the dynamic forms, which reflects the real-time cluster performance, and the cluster partition method is given with the genetic algorithm. Finally, the effectiveness verification of the proposed cluster partition method is carried out with the 14- and 33-bus systems.
\end{abstract}

\section{Introduction}

In recent years, due to the support of national renewable energy policy for sustainable development and people's awareness of green environmental protection, large-scale distributed generations (DGs) such as hydroelectric power generation, photovoltaic, and wind power have been developing rapidly, which brings certain impact on the safe and stable operation and management of the power grid [1-3]. Faced with the challenges of DGs integration, it is urgent to improve the integration regulation and control technology of DGs to ensure stable, safe, and economic operation of the grid and develop green and sustainable renewable DGs.

Nowadays, the general regulation and control methods of DGs are centralized control structure, microgrid mode control structure, and cluster control mode structure [4]. Since the renewable DGs in the remote areas are usually small in capacity, large in number and scattered in geographical distribution, it is very difficult to adopt the centralized regulation and control mode. The cluster mode can make orderly and reasonable group distribution for the scattered DGs, which gives full play to the autonomous characteristics of the cluster and guarantees the safe, effective, and reliable integration of the DGs. Among them, the main application scenarios of DG cluster are system planning and scheduling control, and from the contents published in several works in the literature, the focus of cluster partition is on scheduling control, such as grid partitioning $[5,6]$, voltage control partitioning [7-9], and group regulation and group control $[10,11]$. In [12], a distributed Newton-based voltage control method is presented to realize distributed coordination of PV generation cluster, which can fast respond to voltage mismatch and realize voltage profiles optimization. In [13], a cluster-oriented cooperative control strategy for multiple ac MG clusters is proposed to achieve power sharing among multiple MG clusters, whereas the frequency/voltage of all DGs within each MG cluster can also be regulated. Although the robust distributed cluster methods for DGs have been discussed in the above publications, the reasonable DG 
clusters partition algorithms are the basis for realizing the regulation and control of large-scale DGs, which are not studied in depth in the existing researches. What is more, how to effectively realize the dynamic and real-time DG clusters partition is barely reported in the researches. Compared with other cluster partition problems for power system, the factors needed to consider DGs cluster partition are relatively more, including the electrical distance, the active and reactive power balance, and the flexible resources. How to combine these factors to form the real-time comprehensive index in the expanded dynamic time scenarios is the main difference. In summary, the improvement of the partition method makes DG cluster become an important solution for renewable energy grid integration $[14,15]$ and how to realize the dynamic cluster partition of DGs considering expanded dynamic time scenarios needs to be furtherly studied.

In this article, the influence of internal and external flexible resources is considered in the DG cluster division, and the comprehensive performance indexes with the expanded dynamic time scenario are proposed to realize the dynamic cluster partition. The main contributions of this artice can be summarized as follows:

(1) The active and reactive power balance indexes considering the internal and external flexible resources are proposed, which form the comprehensive index together with the structure index.

(2) The comprehensive index is expanded to the dynamic forms, which reflects the real-time cluster performance, and the cluster partition method is given with the genetic algorithm.

Finally, the effectiveness verification of the proposed comprehensive index is carried out with the 14- and 33-bus system.

\section{Comprehensive Performance Indexes for DG Cluster Partition}

2.1. Electrical Structure Index. In order to reflect the electrical distance of the DG clusters, the concept of modularity index [16] is adopted in this article, which is defined as follows:

$$
\rho=\frac{1}{2 m} \sum_{u} \sum_{v}\left(D_{u v}-\frac{k_{u} k_{v}}{2 m}\right) \delta(u, v),
$$

where $D_{u v}$ denotes the edge weight of the node $u$ and the node $v$, and when two nodes are directly connected, it has $D_{u v}=1$; otherwise, $D_{u v}=0$. The sum of the weights of all edges in the clusters is defined as $m=\sum_{u} \sum_{v} D_{u v} / 2 ; k_{u}=$ $\sum_{v} D_{u v}$ denotes the sum of the all edge weights that are directly connected to the node $u$. As for $\delta(\mathrm{u}, \mathrm{v})$, its value is 1 if two nodes are in the same cluster; otherwise, it is 0 .

In this article, the edge weights of the nodes will be determined based on the electrical distance, which can reflect the tightness of the coupling between the nodes. The specific relationship equation is as follows:

$$
\left\{\begin{array}{l}
\Delta V=S_{\mathrm{VQ}} \Delta \mathrm{Q}, \\
d_{\mathrm{uv}}=\lg \frac{S_{\mathrm{VQ}, \mathrm{vv}}}{S_{\mathrm{VQ}, \mathrm{uv}}},
\end{array}\right.
$$

where $\Delta V$ and $\Delta Q$ are the voltage magnitude and reactive power variation, respectively; $S_{V Q}$ is the sensitivity matrix and obtained by partial differentiation of the node voltage to reactive power; and $S_{V Q, u v}$ is the value of reactive power variation at the node $v$. Considering that the weight relationship between two nodes is also associated with other nodes in the network, let there be a total of $n$ nodes in the clusters, and the electrical distance between two nodes is $L_{u v}$, which is shown as follows:

$L_{u v}=\sqrt{\left(d_{u 1}-d_{v 1}\right)^{2}+\left(d_{u 2}-d_{v 2}\right)^{2}+\cdots+\left(d_{u n}-d_{v n}\right)^{2}}$.

The electrical distance (3) is the quantification of the node voltage coupling degree, and the larger the value, the closer the connection between nodes. Considering the actual situation, the time-varying load demand and the DG output also have an influence on the electrical distance, so the time scenario when the DG output penetration is the highest should be chosen, which means the maximum values of $P_{\text {re }}(t) / P_{\text {load }}(t)$ and $P_{\text {re }}(t)$ are selected to calculate the electrical structure index.

2.2. Active and Reactive Power Balance Indexes. The active power index $\varphi_{P}$ of the cluster is mainly reflected by the ability of the DGs clusters to achieve active power balance, which is defined as follows:

$$
\left\{\begin{array}{l}
P_{i}=\left|\frac{P_{s}(t)_{i}+\Delta P_{G_{i}}(t)+\Delta P_{G_{i}}^{\prime}(t)}{\max \left(P_{s}(t)_{i}+\Delta P_{G_{i}}(t)+\Delta P_{G_{i}}^{\prime}(t)\right)}\right|, \\
j_{P}=\frac{1}{c} \sum_{i=1}^{c} P_{i},
\end{array}\right.
$$

where $P_{s}(t)_{i}$ is the active power output of the DGs in the cluster $i$ at each time period, $\Delta P_{G i}(t)$ is the active power provided by adjustable flexible resources in the cluster $i$, $\Delta P_{G_{i}}^{\prime}(t)$ is the active power provided by external clusters to the cluster $i$, and $P_{i}$ is the active power balance index of the cluster. From equation (4), the active balance index $\varphi_{P}$ of some cluster is obtained under the time scenario with the highest DG output penetration, which indicates the power constraints and source-load balance state of the clusters. Besides, the power support of adjustable flexible resources in external clusters is also considered in (4).

As for the reactive voltage regulation of the cluster, it should have certain regulation capability of maintaining the reactive power balance and the voltage at rated level. When the internal reactive power regulation capacity of the cluster reaches the upper limit, it is also necessary to achieve the reactive power balance of the cluster through the mutual 
support of multiple clusters. Therefore, the reactive power balance index $\varphi_{Q}$ is defined as follows:

$$
\left\{\begin{array}{l}
Q_{i}=\frac{Q_{\text {insup }}(t)+Q_{\text {outsup }}(t)}{Q_{\text {need }}(t)}, \\
j_{Q}=\frac{1}{c} \sum_{i=1}^{c} Q_{i},
\end{array}\right.
$$

where $Q_{i}$ is the ratio of reactive power supply and demand of the cluster, including the maximum reactive power $Q_{\text {insup }}$ provided by the cluster and the reactive power $Q_{\text {outsup }}$ provided by other external clusters; $\varphi_{Q}$ is the reactive power balance index; $c$ is the number of clusters; and $Q_{\text {need }}$ is the reactive power demand within the cluster, and from the literature [17], it is known that in addition to the normal reactive power demand of the cluster, the minimum reactive power required to regulate the node voltage under high penetration conditions is also included.

The sensitivity matrix to reflect the electrical distance of the nodes in the clusters is necessary for the clusters partition, which is proposed in [18]. Besides, the active and reactive power balance in (4) and (5) are also basic factors in the DGs clusters partition. However, the internal and external flexible resources should be considered in the active and reactive power balance indexes. Then, the comprehensive index together with the structure index can be formed, which is shown in the next section.

\section{The Comprehensive Cluster Partition Index considering Expanded Dynamic Time Scenarios}

3.1. The Adjustable Flexibility Resources. This article considers the impact of flexible and adjustable resources in the cluster for active and reactive power support during the cluster partition. The adjustable flexible resources, including electric vehicles, energy storage, and adjustable loads, can flexibly adjust the active power of the cluster according to the demand, which improves the consumption of DGs. The following section will analyze the regulation characteristics of the above adjustable flexible resources.

The load characteristics of the adjustable electric vehicle are known from the literature [19]. Let $P_{\text {evi,t }}$ be the power of the $i$ th electric vehicle at the time $t$, and its value is the sum of the rated power and the regulated power $\Delta P_{e v i, t}$. If there are $N$ electric vehicles involved in power regulation, the expression of the total power regulation is as follows:

$$
\Delta P_{\mathrm{ev}, \mathrm{t}}=\sum_{\mathrm{i}=1}^{N} \Delta P_{\mathrm{evi}, t} .
$$

The constraint is that the power of the electric vehicle needs to be greater than or equal to its maximum discharge power and less than or equal to its maximum charge power.

In the adjustable power of the energy storage system, if the power emitted by the renewable energy in the cluster $i$ is $P_{i}(t)$, the capacity state of the energy storage is $E_{t}$, and the power demanded by the load is $P_{\text {load }}(t)$, then it has $\Delta P(t)=$ $P_{i}(t)-P_{\text {load }}(t)$. Then the integral of $\Delta P(t)$ is then energy written as $\Delta E_{s}(t)$, and its positive and negative sign represents the charge and discharge state of the energy storage system, respectively. Hence, in the $\Delta \tau$ time interval, the storage system needs to store (or release) the energy $E(t)$ and the expression is as follows:

$$
E(t)=\int_{t}^{t+\Delta \tau} \Delta E_{s}(t) \mathrm{d} t .
$$

Therefore, the expression of the capacity state of the energy storage system is as follows:

$$
E_{t}=E_{0}+\mathrm{E}(t)
$$

where $E_{0}$ is the initial state capacity of the energy storage system.

The relationship of the power that can be provided by the adjustable load is expressed as follows:

$$
\Delta P_{a l}(t)=\gamma_{a l, t} P_{a l}(t)
$$

where $\gamma_{a l, t}$ denotes the proportion of the adjustable load participating in the demand response and $P_{a l}(t)$ denotes the total power of the adjustable load.

In addition to the above regulation means, the conventional available power regulation methods in the distribution network are still the adjustable small hydropower and standby thermal power units, and the ability to adapt the active power imbalance of the system is achieved by involving some units with adjustable space in the power regulation response of the system. Therefore, for the conventional adjustable units within the cluster, the power output at the time can be set as $P_{G, t}$. By analyzing the different forms of adjustable flexible resources mentioned above, the final sum of adjustable power within the cluster can be obtained as follows:

$$
\Delta P_{G_{i}}(t)=\sum_{e v, E, a l,}\left(\Delta P_{e v}(t)+\Delta P_{E}(t)+\Delta P_{a l}(t)+\Delta P_{G}(t)\right) .
$$

3.2. Comprehensive Index considering Expanded Dynamic Time Scenarios. Based on the various types of indexes mentioned above, the comprehensive index is obtained as follows:

$$
\gamma=\lambda_{1} \rho+\lambda_{2} j_{P}+\lambda_{3} j_{Q}
$$

where $\lambda_{1}, \lambda_{2}$, and $\lambda_{3}$ are the weight of each index. The cluster comprehensive performance assessment index shown in (11) represents the highest penetration time of a day. If the time is divided equally into $N$ intervals of 24 hours a day, there must be a composite index $\gamma_{t}$ of high penetration in each interval $[t, t+\Delta \tau]$. From the mathematical differentiation method, the integrated performance index function can be obtained by computer fitting as follows:

$$
f(t)=\gamma(t)
$$


The function $f(t)$ reflects the cluster partition results, and if the cluster partition meets expectation, the active and reactive power balance index in the function has no fluctuation, which means the derivative of the function tends to be smooth. Hence, if the ideal value of the comprehensive performance index is taken as the starting value of the horizontal coordinates and the absolute value of the function integration is taken in the time interval, then the size of the obtained area $S$ reflects the cluster division. The smaller $S$ is, the better the partition will be. The expression of the cluster classification $S$ is shown as follows:

$$
S=\int_{0}^{T} f(t) \mathrm{d} t
$$

For the comprehensive performance index function $f(t)$ defined on the interval $[0, T]$, and since it meets the Dirichlet sufficient conditions, the period even extension can be conducted to $f(t)$ to extend its domain to become $[-T, T]$. Then it can be expanded into Fourier series as follows:

$$
\left\{\begin{array}{l}
f(t)=\frac{a_{0}}{2}+\sum_{n=1}^{\infty}\left(a_{n} \cos n \omega_{0} t\right), \\
a_{n}=\frac{2}{T} \int_{0}^{T} f(t) \cos n \omega_{0} t \mathrm{~d} t, n=0,1,2,3, L,
\end{array}\right.
$$

where $a_{n}$ can be obtained by the divisional integration method as follows:

$$
a_{n}=\frac{f(T) \sin n \omega_{0} T}{(n \pi / 2-s)}
$$

where the value $s$ is

$$
s=\frac{\ln n+\mathrm{R}}{\omega_{0}} \int_{0}^{T} \sin ^{2} n \omega_{0} t \mathrm{~d} t
$$

where $\omega_{0}=\pi / 2 T$ and $R=0.577218$ is the Euler constant. Therefore, the final Fourier series of the expansion of the function on the interval $[0, T]$ is obtained as follows:

$$
f(t)=\frac{1}{T} \int_{0}^{T} f(t) \mathrm{d} t+\sum_{\mathrm{n}=1}^{\infty}\left(\frac{f(T) \sin n \omega_{0} T}{n \pi / 2-s} \cos n \omega_{0} t\right)
$$

where $n=1,2,3, \ldots$ As for $\int_{0}^{T} f(t) \mathrm{d} t$, the approximation method can be used and obtains the following:

$$
\int_{0}^{T} f(t) \mathrm{d} t=\frac{T}{N} \sum_{\mathrm{N}=1}^{\infty} \gamma_{\mathrm{t}, \mathrm{N}}=S_{0}
$$

If we take a finite number of values $N$ and an approximation of $S_{0}$ can be obtained, then the final function can be expressed as follows:

$$
f(t)=\frac{S_{0}}{T}+\sum_{n=1}^{\infty}\left(\frac{f(T) \sin n \omega_{0} T}{n \pi / 2-s} \cos n \omega_{0} t\right) .
$$

3.3. Improved Cluster Partition Algorithm. Based on the comprehensive index considering expanded dynamic time scenarios, an improved genetic algorithm is adopted in this article. The genetic algorithm applied to cluster partition usually takes the integrated performance index as the fitness function and the result of cluster partition as the solution problem. For the several complex DGs in clusters, the cluster partition algorithm is improved in this article and the time complexity of the genetic algorithm can be low, while the large number of nodes does not significantly increase the computation time.

In this article, the binary is encoded based on the adjacency matrix of the network. Not only does this encoding method make the nodes ensure the connectivity performance, but also it reduces the search range of the genetic algorithm and reduces the search time. At the same time, this encoding method does not have the process of node merging when performing cluster partition, which speeds up the iterative process of the probabilistic mechanism and enhances the search capability for irregular clusters. The illustration of the adjacency matrix is shown in Figure 1.

The adjacency matrix can represent the connectivity of nodes in a cluster network, which includes only elements 0 and 1 , and 1 indicates that the nodes are connected and 0 indicates that the nodes are not connected. The genetic algorithm randomly modifies the elements of the matrix according to the optimization when searching the encoding. The new adjacency matrix will be formed after the search is completed, which represents a new cluster partition.

In order to improve the iteration efficiency of the genetic algorithm, this article adopts the adaptive idea, while the crossover rate and variation rate are dynamically adjusted according to the adaptability of the solution. The principle of adjustment is as follows: for individuals with high fitness, the crossover and variation process are performed, while the variation probability is increased, and the crossover probability is decreased to enhance the local search ability of the algorithm. For individuals with low fitness, the crossover and variation rates are assigned according to the iteration state. As the number of iterations increases, the results of cluster partition tend to be closer to the optimal value. The specific values are adopted from the method proposed by Srinivas and the specific formula is as follows:

$$
\begin{aligned}
& p_{c}= \begin{cases}p_{c, \max }-\left(\frac{p_{c, \max }-p_{c, \min }}{I_{\max }}\right) I, & f>f_{\mathrm{avg}}, \\
p_{c, \max } & f \leq f_{\mathrm{avg}},\end{cases} \\
& p_{m}= \begin{cases}p_{m, \min }-\left(\frac{p_{m, \max }-p_{m, \min }}{I_{\max }}\right) I, & f^{\prime}>f_{\mathrm{avg}}, \\
p_{c, \max } & f^{\prime} \leq f_{\mathrm{avg}},\end{cases}
\end{aligned}
$$

where $p_{c}$ and $p_{m}$ are the crossover and variation probabilities, respectively; $p_{c, \max }, p_{c, \text { min }}$, and $p_{m, \max }, p_{m, \text { min }}$ are the 


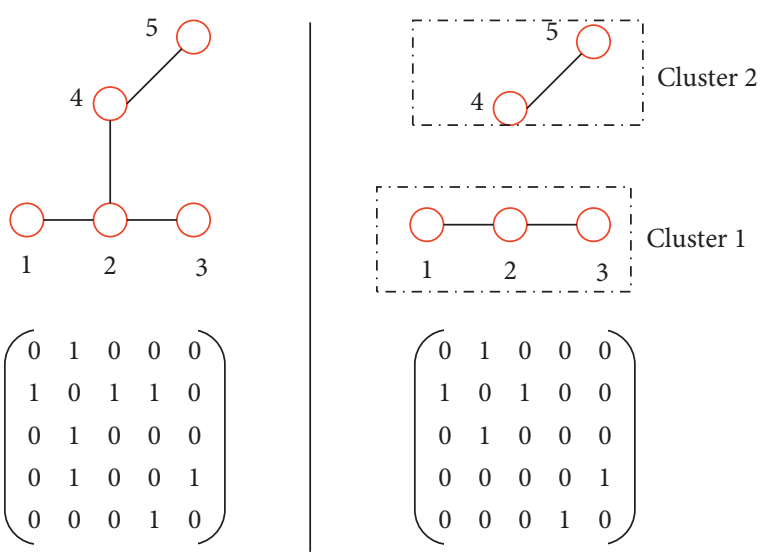

FIgURE 1: The illustration of the adjacency matrix.

maximum and minimum values of the crossover and variation probabilities, respectively; $I$ are the number of iterations; $f$ represent the fitness value of the individual undergoing the variation, and $f$ is the maximum fitness value of the two individuals undergoing the crossover; and $f_{\text {avg }}$ is the average fitness value.

\section{Case Study}

In this article, the 14-bus system is selected as the case to verify the effectiveness of the proposed cluster partition method and the comprehensive dynamic performance function. The network topology of the 14-bus system is shown in Figure 2.

The network shown in Figure 2 has a total of 14 nodes, while nodes $1,2,3,6,7$, and 8 are conventional power nodes and do not participate in cluster partitioning. The detailed parameters of the network are presented in the literature [20]. To verify the feasibility of the cluster partition method based on the comprehensive performance index function proposed in this article, the time scenario of the DGs in the case with high penetration is selected, and the electrical sensitivity results between the nodes are calculated shown in Table 1 and the electrical distances are obtained in Table 2.

4.1. Active and Reactive Power Balance Index Results. The genetic algorithm adopted in this article is applied to cluster partition. The number of populations $N$ is 30 and the maximum number of iterations of genetics $I_{\max }$ is 40 . Besides, the probability of variation $p_{m}$ is $(0.2,0.7)$ and the probability of crossover $p_{c}$ is also $(0.2,0.7)$. The tracing of the optimal solution of cluster partition is calculated through several iterations, and the final trend is obtained in Figure 3.

The results show that after the iteration reaches 15 times, the solution of modularity is optimal, at which time the value of modularity is 0.1435 . According to the analysis of the adjacency matrix at the final solution, the results of cluster partition can be obtained as nodes $9,10,11,12,13$, and 14 are grouped into one cluster, and nodes 4 and 5 are divided into one cluster, respectively.

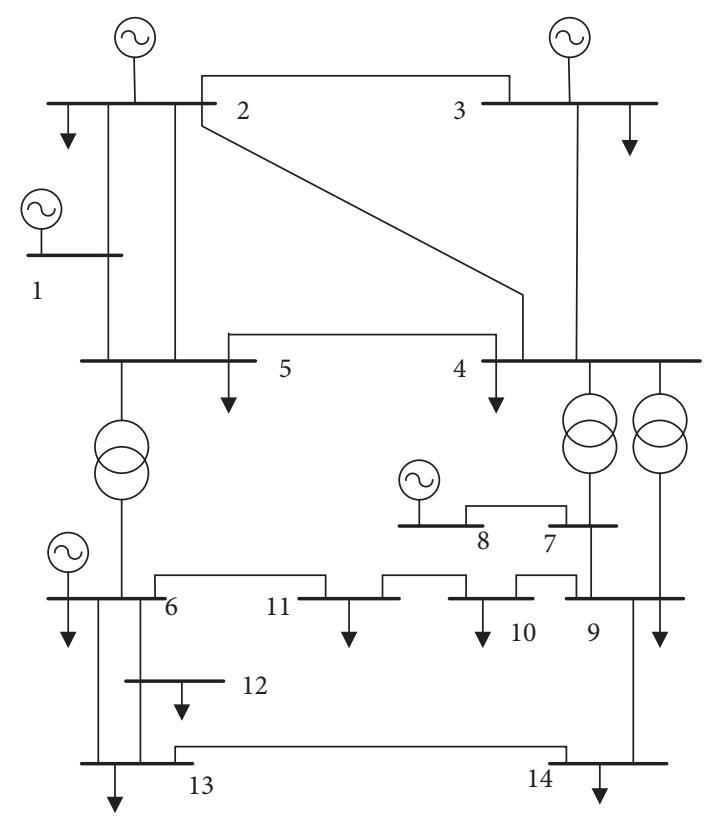

Figure 2: The 14-bus system topology.

According to the 14-bus system data and the genetic algorithm cluster partition results, the power output of each node in a 24-hour day can be obtained as shown in Figure 4 . To verify the feasibility of the adjustable resources for the reactive and active power balance in the intracluster partition method, the flexibility resources are configured in each cluster according to the demand of the system and the power that can be provided by the adjustable resources is shown in Table 3. It is seen that most adjustable flexibility resources are standby resources within the clusters and are rarely connected to the distribution network, and the power output remains basically unchanged under the time change.

To facilitate the derivation for the subsequent comprehensive performance function, the calculation of the coefficients needs to take a finite number. Hence, in the $24 \mathrm{~h}$ time interval, the nine moments, $0,3,6,9,12,15,18$, 21 , and 24 , are selected as the time scenario for the case study. According to the calculation of (4) and (6), the active and reactive power balance indexes of the network structure in the different time scenes are obtained are shown in Table 4.

Since the degree of variation in functional metrics is generally smaller than that in structural metrics, the final weight division combination is chosen as $\lambda 1=0.4, \lambda 2=0.3$, and $\lambda 3=0.3$ to analyze the comprehensive performance metrics of cluster division.

4.2. Comprehensive Performance Index Function. In this case, the key values of $N$ and $n$ are selected as 9 and 5 , and the comprehensive performance index function can be shown as (21), while the function simulation curve graph is given in Figure 5. 
TABLE 1: The electrical sensitivity results between the nodes.

\begin{tabular}{lcccccccc}
\hline Node & 4 & 5 & 9 & 10 & 11 & 12 & 13 \\
\hline 4 & 0.0727 & 0.0072 & 0.0205 & 0.0052 & 0.0009 & 0.0003 & 0.0012 & 0.0066 \\
5 & 0.0445 & 0.0119 & 0.0125 & 0.0032 & 0.0006 & 0.0002 & 0.0007 & 0.0040 \\
9 & 0.0311 & 0.0031 & 0.1321 & 0.0343 & 0.0061 & 0.0015 & 0.0077 & 0.0398 \\
10 & 0.0255 & 0.0025 & 0.1092 & 0.0556 & 0.0099 & 0.0012 & 0.0063 & 0.0327 \\
11 & 0.0126 & 0.0013 & 0.0541 & 0.0276 & 0.0180 & 0.0006 & 0.0031 & 0.0161 \\
12 & 0.0020 & 0.0002 & 0.0089 & 0.0024 & 0.0004 & 0.0318 & 0.0229 & 0.0143 \\
13 & 0.0043 & 0.0004 & 00184 & 0.0048 & 0.0008 & 0.0094 & 0.0464 & 0.0295 \\
14 & 0.0197 & 0.0020 & 0.0847 & 0.0221 & 0.0039 & 0.0053 & 0.0257 & 0.1354 \\
\hline
\end{tabular}

TABle 2: The electrical distance results between the nodes.

\begin{tabular}{lcccccccc}
\hline Node & 4 & 5 & 9 & 10 & 11 & 12 & 13 \\
\hline 4 & 0.0000 & 0.5897 & 2.0089 & 2.1103 & 1.9845 & 3.3287 & 2.8758 & 2.6190 \\
5 & 0.5897 & 0.0000 & 2.5170 & 2.6023 & 2.3871 & 3.5326 & 3.1847 & 3.0975 \\
9 & 2.0089 & 2.5170 & 0.0000 & 0.3681 & 1.0703 & 3.0207 & 2.2429 & 1.0219 \\
10 & 2.1103 & 2.6023 & 0.3681 & 0.0000 & 0.8411 & 3.1440 & 2.3891 \\
11 & 1.9845 & 2.3871 & 1.0703 & 0.8411 & 0.0000 & 3.0826 & 2.4460 & 1.2362 \\
12 & 3.3287 & 3.5326 & 3.0207 & 3.1440 & 3.0826 & 0.0000 & 0.9772 \\
13 & 2.8758 & 3.1847 & 2.2429 & 2.3891 & 2.4460 & 0.9772 & 0.0000 \\
14 & 2.6190 & 3.0975 & 1.0219 & 1.2362 & 1.7763 & 2.5324 & 1.6864 & 0.00000 \\
\hline
\end{tabular}

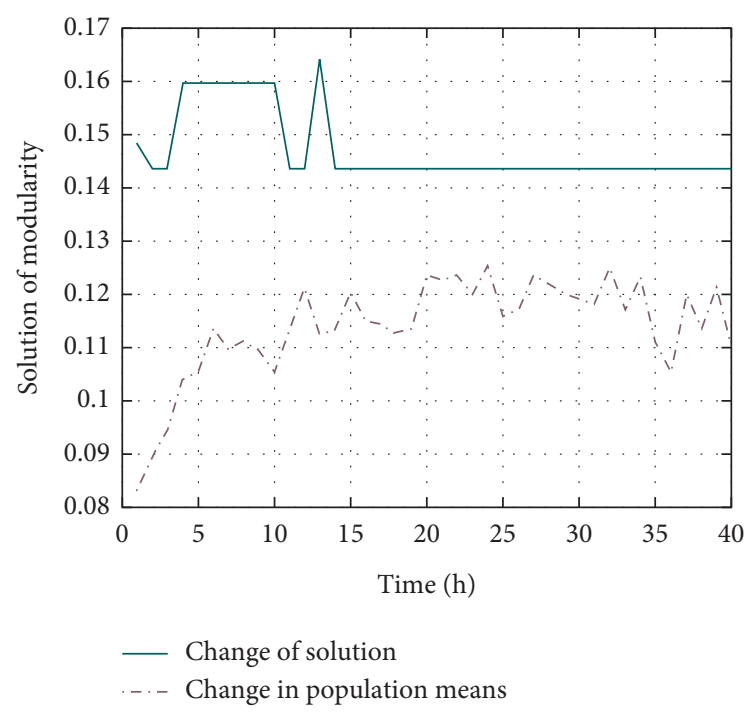

FIgURE 3: Variation of modularity of genetic algorithm solutions.

$$
\begin{aligned}
\gamma(t)= & 0.8793-0.0166 \cos \left(\frac{\pi}{24} t\right)-0.00572 \cos \left(\frac{\pi}{8} t\right) \\
& -0.00442 \cos \left(\frac{5 \pi}{24} t\right) t \hat{\mathrm{I}}[0,24] .
\end{aligned}
$$

The results show that the comprehensive performance function here is expected as derived through mathematical calculation. The function is integrated into the horizontal coordinates over the time interval $[0,24]$ with absolute values, and the final obtained area $\Delta S^{\prime}$ is 7.25. In the ideal case, the values of the modularity index, reactive power, and active power balance index should be 1, and the optimal value of the comprehensive performance index is also 1 .
Then, the function curve should be a straight line, and its integral area over the specified time interval should be zero. The comparison of the integral area shows that the results of the comprehensive performance function based on the improved genetic algorithm for the cluster partition do not differ much from the results of the optimal solution, which indicates that the improved genetic algorithm for the cluster partition has good results during the expanded dynamic time scenarios.

4.3. Comparison of Different Methods. In order to illustrate the effectiveness and rationality of the method proposed in this article, the cluster delineation method in [21] is selected 


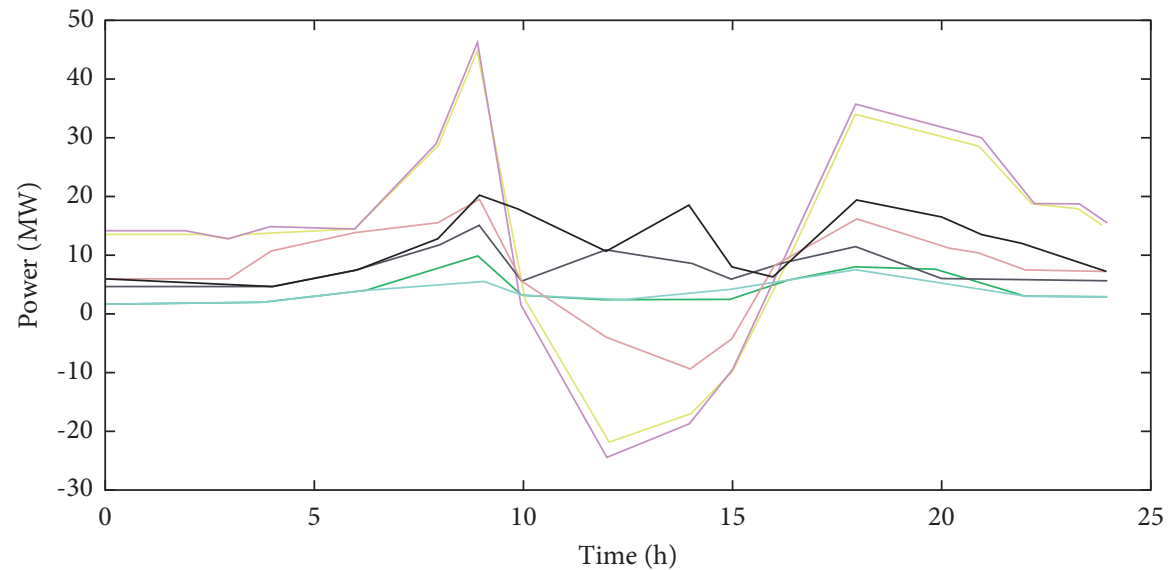

$\begin{array}{rr}\text { Node4 } & \text { Node11 } \\ \text { Node5 } & \text { Node } 2 \\ - \text { Node9 } & \text { Node13 } \\ - \text { Node10 } & \text { Node14 }\end{array}$

FIgURE 4: Output curve of each node in 24 hours.

TABLE 3: Flexible resource output power within each cluster.

\begin{tabular}{lccc}
\hline Cluster & Node & Active power/MW & Reactive power/MVar \\
\hline 1 & 4 & 4.0936 & 0.3354 \\
2 & 5 & 0.6536 & 0.1347 \\
3 & $9,10,11,12,13,14$ & 6.579 & 3.1476 \\
\hline
\end{tabular}

TABLE 4: Index of active and reactive power balance at different time periods.

\begin{tabular}{lccccccccc}
\hline Time & 0 & 3 & 6 & 9 & 12 & 15 & 18 & 21 & 24 \\
\hline$\varphi_{Q}$ & 1.40 & 1.47 & 1.17 & 0.946 & 2.43 & 6.92 & 0.989 & 1.14 & 1.43 \\
$\varphi_{P}$ & 1.25 & 1.31 & 1.06 & 0.736 & 1.00 & 1.00 & 0.802 & 0.738 & 1.40 \\
\hline
\end{tabular}

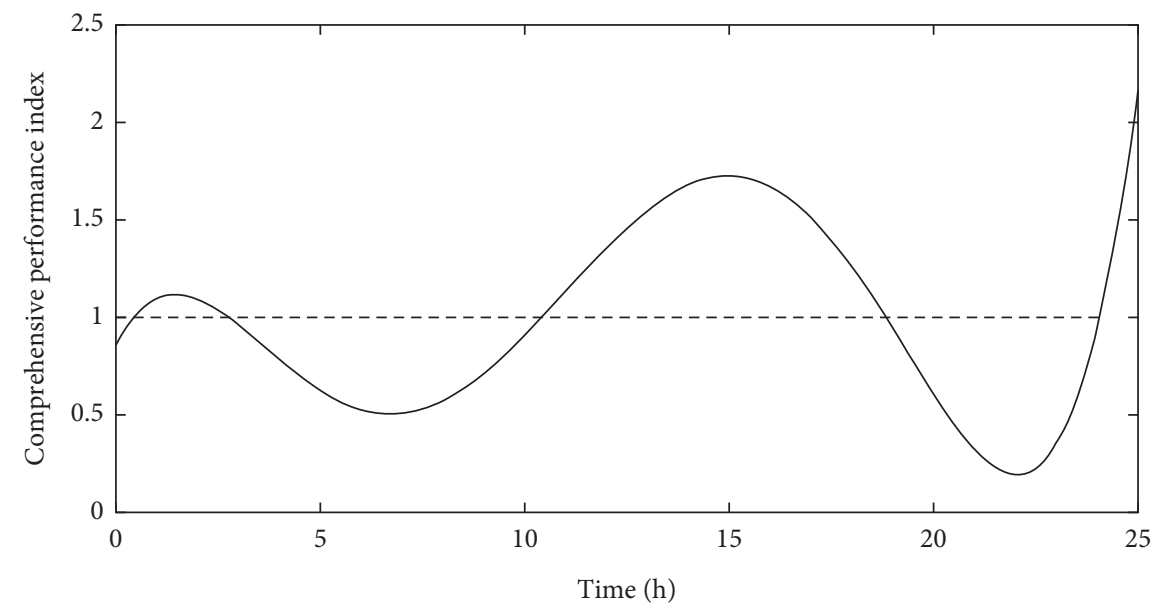

FIGURE 5: Comprehensive performance index simulation function graph.

and compared, and the results are shown in Table 5. The modularity index values of the improved and compared methods are 0.1435 and 0.1340 , respectively, which show that the compared method has a smaller modularity index value, and this indicates that the cluster partition effect is slightly worse than that of the improved genetic algorithm. The reason is that the clusters are classified according to the number of similar elements and by dividing the nodes into 
TABLE 5: Comparison results with different methods.

\begin{tabular}{lcc}
\hline Cluster & \multicolumn{2}{c}{ Different methods } \\
\hline 1 & Improved method is this article & Methods in [27] \\
2 & 4 & 4,5 \\
2 & 5 & 9,10 \\
\hline
\end{tabular}

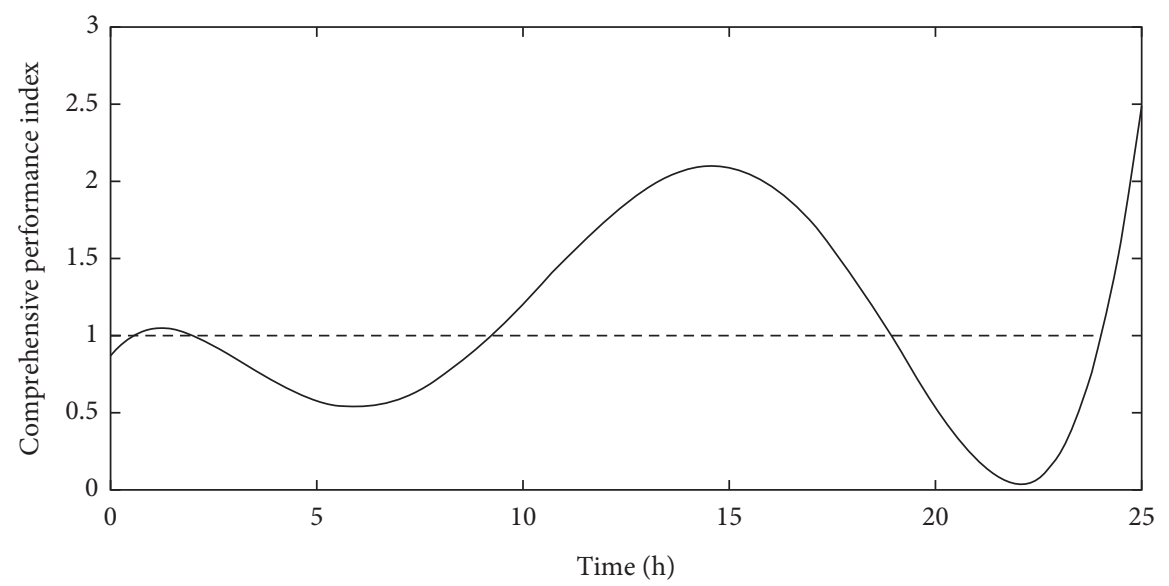

FIGURE 6: Integrated performance index function graph of cluster algorithm.

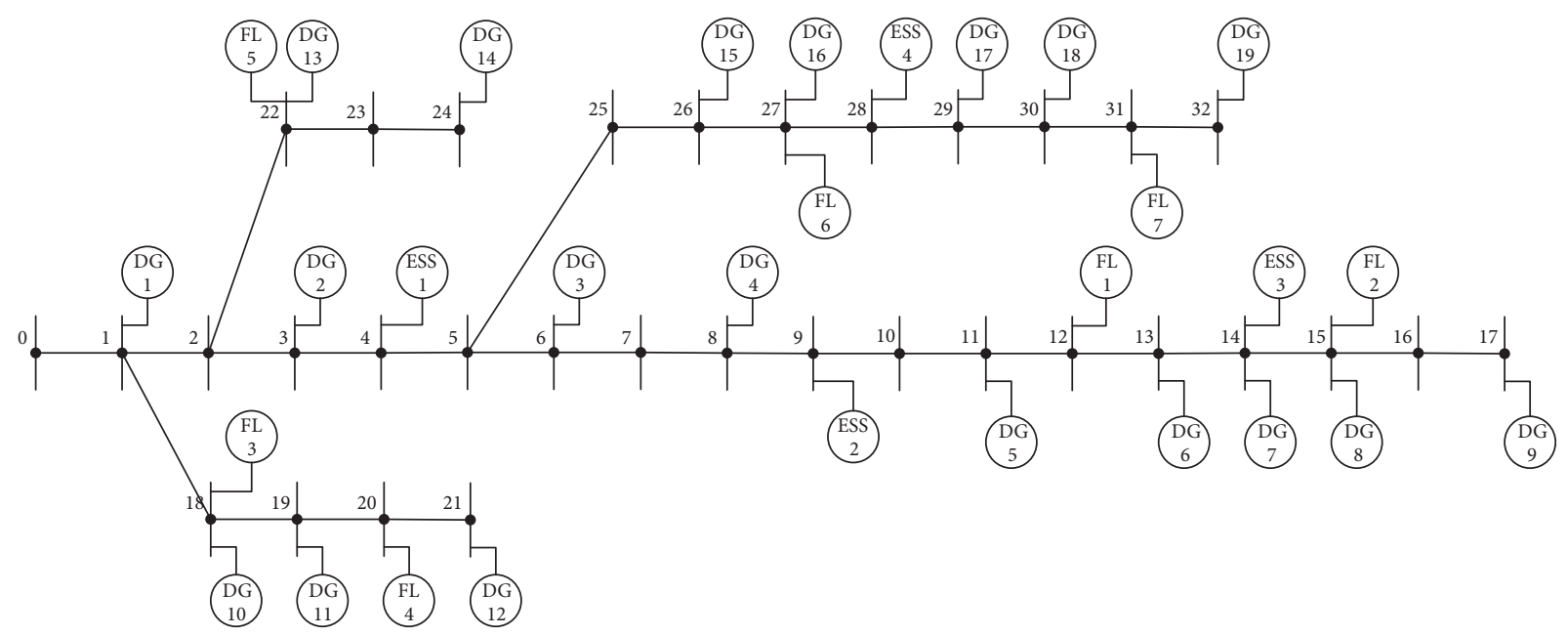

FIGURE 7: The 33-bus system grid topology.

classes with the compared method. In order to average the final results, the compared method combines nodes 9 and 10 into the same cluster, resulting in low overall modularity. However, the improved algorithm adopts crossover and variation probabilities for iterative operations to solve the problem, which belongs to global optimization and can take the overall optimization among clusters into account.

In this article, the integrated performance index function is used as the basis for partition, and the results by different methods are used to compare the indexes. From the above- analyzed results, the integrated performance index function curve is shown in Figure 6. The area $\Delta S$ " is 9.15 obtained by integrating the function curve with a straight line $y=1$ as the horizontal coordinate under the compared method. However, under the proposed method, the area $\Delta S^{\prime}$ is $7.25 \mathrm{ob}-$ tained by the improved genetic algorithm. It is seen that the difference between the two methods is large, which means that the improved genetic algorithm is more globally superior to the compared method. The comparison of the area of the comprehensive performance index function curve also 


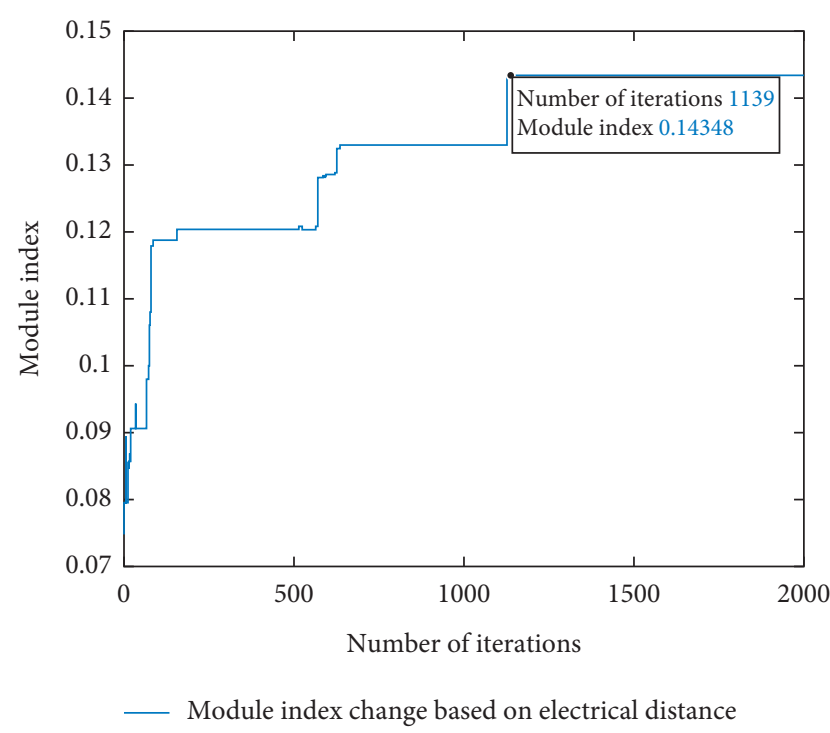

FIgURe 8: Modularity variation by genetic algorithm solution.

verifies the advantages of the genetic algorithm, which further proves the feasibility of the comprehensive performance index function partition method.

4.4. Case Study of the 33-Bus System. To further illustrate the effectiveness of the proposed cluster partition index in dynamic time-scale, the case study of the 33-bus system is studied in this section. The network topology of the 33-bus system grid topology is shown in Figure 7.

The network has a total of 33 nodes, and to simulate the actual scene of DGs scattered in the network, nodes $1,3,6,8$, $11,13,14,15,17,18,19,21,22,24,26,27,29,30$, and 32 are inserted into the DGs (all assumed to be photovoltaic here). Besides, the flexible loads are inserted at nodes 12, 15, 18, 20, 22,27 , and 31 . In addition, the energy storage device is inserted at nodes $4,9,14$, and 28 . In order to verify the feasibility of the cluster division method, the time scenario of distributed power supply with high permeability is selected. In this case, the number of populations $N$ is 200 and the maximum number of iterations of genetics $I_{\max }$ is 2000 . Besides, the probability of variation $p_{m}$ is $(0.2,0.7)$ and the probability of crossover $p_{c}$ is also $(0.2,0.7)$. Through several iterations, the optimal solution of cluster division is tracked, and the change trend is finally obtained, as shown in Figure 8 . The results show that after the number of iterations reaches 1139 , the solution of modularity reaches the optimal value, and the value of modularity is 0.14348 . According to the analysis of the adjacency matrix in the final solution, it can be concluded that nodes $1,2,3,4,22,23$, and 24 are cluster 1 , nodes $5,6,7,8,9$, and 10 are cluster 2 , and nodes $11,12,13,14,15,16$, and 17 are cluster 3 . Nodes 18, 19, 20, $21,25,26,27,28,29,30,31$, and 32 are cluster 4 , which further indicates the availability of the proposed cluster partition method in this article.

\section{Conclusion}

This article conducts more comprehensive research on DG cluster partition expanding from a static moment to dynamic time scenarios. Firstly, the active and reactive power balance indexes considering the internal and external flexible resources are proposed, which forms the comprehensive index together with the structure index. Then, the comprehensive index is expanded to the dynamic forms, which reflects the real-time cluster performance, and the cluster partition method is given with the genetic algorithm. It should be noticed that the proposed method can also be applied in other large-scale industrial systems such as pipeline systems $[22,23]$, while the attention points are that a proper comprehensive index with the combination of different single evaluation index should be given. Finally, the effectiveness verification of the proposed comprehensive index is carried out with the 14- and 33-bus system.

\section{Data Availability}

The data that support the findings of this study are available from the corresponding author upon reasonable request.

\section{Conflicts of Interest}

The authors declare that they have no conflicts of interest.

\section{Acknowledgments}

This research was supported by the Science and Technology Project of State Grid Corporation of China (52153220000U).

\section{References}

[1] B. Yang, L. Yu, Y. Chen et al., "Modelling, applications, and evaluations of optimal sizing and placement of distributed generations: A critical state-of-the-art survey," International Journal of Energy Research, vol. 45, no. 3, pp. 3615-3642, 2021.

[2] Z. Liu, S. Miao, Z. Fan, J. Liu, and Q. Tu, "Improved power flow control strategy of the hybrid AC/DC microgrid based on VSM," IET Generation, Transmission \& Distribution, vol. 13, no. 1, pp. 81-91, 2019.

[3] A. El-Ela, R. A. El-Sehiemy, E. S. Ali, and A. M. Kinawy, "Minimization of voltage fluctuation resulted from renewable energy sources uncertainty in distribution systems," IET Generation, Transmission \& Distribution, vol. 13, no. 12, pp. 2339-2351, 2019.

[4] P. Wirasanti, E. Ortjohann, M. Hoppe, H. Saffour, S. Leksawat, and D. Morton, "Automated active distribution network with multi-level cluster control approach," in Proceedings of the IECON 2013-39th Annual Conference of the IEEE Industrial Electronics Society, pp. 1980-1985, IEEE, Vienna, Austria, November 2013.

[5] M. Biserica, G. Foggia, E. Chanzy, and J. C. Passelergue, "Network partition for coordinated control in active distribution networks," in Proceedings of the IEEE Grenoble Conference, pp. 1-5, Grenoble, France, June 2013. 
[6] T. N. Pread, K. Uhlen, and D. Nordgard, "Clustering distributed generation using the instantaneous Euclidean distance in polar coordinates," in Proceedings of the 4th IEEE PES Innovative Smart Grid Technologies (ISGT) Europe, Lyngby, Denmark, October 2013.

[7] S. Huang, Q. Wu, J. Zhao, and W. Liao, "Distributed optimal voltage control for VSC-HVDC connected large-scale wind farm cluster based on analytical target cascading method," IEEE Transactions on Sustainable Energy, vol. 11, no. 4, pp. 2152-2161, 2020.

[8] J. Lai, X. Lu, Z. Dong, and S. Cheng, "Resilient distributed multiagent control for AC microgrid networks subject to disturbances," IEEE Transactions on Systems, Man, and Cybernetics: Systems, early access, vol. 52, no. 1, 2021.

[9] J. Lai and X. Lu, "Communication constraints for distributed secondary control of heterogenous microgrids: A brief survey," in Proceedings of the IEEE/IAS 57th Industrial and Commercial Power Systems Technical Conference, April 2021.

[10] Y. Nie, W. Liu, and C. Xu, "Comprehensive evaluation of active power control effect of wind power cluster based on AHP and fuzzy evaluation method," in Proceedings of the IEEE Sustainable Power and Energy Conference (iSPEC), IEEE, Beijing, China, November 2019.

[11] L. Ye, C. Zhang, Y. Tang et al., "Hierarchical model predictive control strategy based on dynamic active power dispatch for wind power cluster integration," IEEE Transactions on Power Systems, vol. 34, no. 6, pp. 4617-4629, 2019.

[12] Z. Wang, L. Ge, J. Ding, and W. Gu, "Distributed Newtonbased voltage control method for high-penetration PV generation cluster in active distribution networks," IET Renewable Power Generation, vol. 14, no. 11, pp. 1904-1911, 2020.

[13] J. Lai, X. Lu, X. Yu, and A. Monti, "Cluster-oriented distributed cooperative control for multiple ac microgrids," IEEE Transactions on Industrial Informatics, vol. 15, no. 11, pp. 5906-5918, 2019.

[14] M. Ding, H. Fang, and B. Rui, "Optimal siting and sizing of distributed PV-storage in distribution network based on cluster partition," Proceedings of the CSEE, vol. 39, no. 8, pp. 2187-2201, 2019.

[15] S. Leksawat, A. Schmelter, E. Ortjohann et al., "Demonstration of cluster-based power system automation for future smart grids," in Proceedings of the IEEE International Energy Conference (ENERGYCON), pp. 1-6, IEEE, Leuven, Belgium, April 2016.

[16] R. Bi, X. Liu, and M. Ding, "Renewable energy generation cluster partition method aiming at improving accommodation capacity," Proceedings of the CSEE, vol. 39, no. 22, pp. 6583-6591, 2019.

[17] M. Ding, X. Liu, and R. Bi, "Method for cluster partition of high-penetration distributed generation based on comprehensive performance index," Automation of Electric Power Systems, vol. 42, no. 15, pp. 47-52, 2018.

[18] D. Ma, X. Hu, H. Zhang, Q. Sun, and X. Xie, "A hierarchical event detection method based on spectral theory of multidimensional matrix for power system," IEEE Transactions on Systems, Man, and Cybernetics: Systems, vol. 51, no. 4, pp. 2173-2186, 2021.

[19] H. Tian, W. Liu, and F. Wang, "Multi-objective optimal method of source-load peaking regulation based on participation of loads," Power System and Clean Energy, vol. 34, no. 11, pp. 32-38, 2018.

[20] B. Zhang, S. Chen, and Z. Yan, Advanced Power Network Analysis, Tsinghua University Press, Beijing, China, 2007.
[21] H. Chen, Y. Yun, and W. Xing, “A novel strategy of network partitioning for load node in power systems using spectral clustering," Power System Protection and Control, vol. 41, no. 12, pp. 47-53, 2013.

[22] X. Hu, H. Zhang, D. Ma, and R. Wang, "Hierarchical pressure data recovery for pipeline network via generative adversarial networks," IEEE Transactions on Automation Science and Engineering, Lyon, France, 2021.

[23] X. Hu, H. Zhang, and D. Ma, "A tnGAN-based leak detection method for pipeline network considering incomplete sensor data," IEEE Transactions on Instrumentation and Measurement, vol. 70, Article ID 3510610, 2021. 